EPJ Web of Conferences 28, 04002 (2012)

DOI: 10.1051/epjconf/20122804002

(C) Owned by the authors, published by EDP Sciences, 2012

\title{
CKM angle measurements and the search for CP violation in charm
}

\author{
Malcolm John ${ }^{\mathrm{a}}$, on behalf of the LHCb collaboration \\ Department of Physics, University of Oxford, Oxford, United Kingdom
}

\begin{abstract}
This contribution reports on recent $\mathrm{LHCb}$ achievements in the pursuit of CKM triangle measurements and probes of $C P$ violation in the charm system. These results are based on the 2010 dataset or, in some cases, preliminary results using the data collected by summer 2011.
\end{abstract}

\section{Introduction}

A fundamental feature of the Standard Model and its three quark generations is that all hadronic $C P$ violation phenomena are the result of a single phase in the CKM quarkmixing matrix [1]. It is well known that due to the unitarity of this matrix, several triangle relations can be formed. One relation that is is readily applicable to $B$ mesons is

$$
0=1+\frac{V_{t b}^{*} V_{t d}}{V_{c b}^{*} V_{c d}}+\frac{V_{u b}^{*} V_{u d}}{V_{c b}^{*} V_{c d}} .
$$

This equation defines a triangle of similar height and width and hence predicts large $C P$ violation in the $B$ system. This is well established $[3,4]$ though one of the three internal angles, $\gamma=-\arg \frac{V_{u d} V_{u b}^{*}}{V_{c d} V_{c b}^{*}}$ remains poorly constrained. The triangle relation relevant to the charm sector is

$$
0=1+\frac{V_{u b}^{*} V_{c b}}{V_{u s}^{*} V_{c s}}+\frac{V_{u d}^{*} V_{c d}}{V_{u s}^{*} V_{c s}} .
$$

which forms a flatter triangle than that of the $B$-system. This flatness is synonymous with an expectation of small $C P$ violation in charm decays.

In the understanding of the CKM paradigm, a detailed examination of both these triangles is vital. In the $B$ system, where $C P$ violation is established, the focus is on evermore precise measurements the triangle metrology where deviations from internal consistency would indicate new physics. With two of these angles well-measured $(\leq 5 \%$, see [5] for useful summaries) LHCb is currently focussed on pursuing the third angle, $\gamma$. Whilst sensitivity to $\gamma$ is not yet possible, Sec. 2 reports the status of several key measurements in this area.

A similar justification holds in charm physics where new-physics couplings to up-type quarks may be uniquely

\footnotetext{
a e-mail: malcolm.john@physics.ox.ac.uk
}

probed. However, the most immediate goal is to establish the existence of $C P$ violation in the charm sector. Sec. 3 reports the status of the searches for $C P$ violation with these decays.

The $\mathrm{LHCb}$ detector [6] takes advantage of the high $b \bar{b}$ and $c \bar{c}$ cross sections at the Large Hadron Collider to collect unprecedented samples of heavy meson decays. It has a spectrometer design instrumenting the pseudorapidity range $2<\eta<5$ of the proton-proton collisions. Critical for these analyses is the tracking system which achieves a momentum resolution of $0.4-0.6 \%$ in the range $5-$ $100 \mathrm{GeV} / c$. A silicon microstrip vertex detector is mounted around the collision region and provides clear separation of $B$ and $D$ decay vertices away from the primary collision vertex. $\mathrm{LHCb}$ benefits from two ring-imaging Cherenkov $(\mathrm{RICH})$ counters with three radiating media: aerogel, $C_{4} F_{10}$ and $\mathrm{CF}_{4}$. These detectors provide dedicated particle identification (PID), vital for the hadronic physics program.

\section{CKM angle measurements}

This section concentrates on the development of modes that have sensitivity to $\gamma$ at $\mathrm{LHCb}$.

\section{$2.1 B^{-} \rightarrow\left[\pi^{-} K^{+}\right]_{D} K^{-}$}

Of vital importance to the extraction of $\gamma$ are measurements of charge asymmetry in $B^{ \pm} \rightarrow D K^{ \pm}$decays where the $D$ may be a $D^{0}$ or a $\bar{D}^{0}$. In this case, the amplitude for the $B^{-} \rightarrow D^{0} K^{-}$contribution is proportional to $V_{c b}$ whilst the $B^{-} \rightarrow \bar{D}^{0} K^{-}$amplitude depends on $V_{u b}$. The interference of these two processes gives sensitivity to $\gamma$ and hence may exhibit direct $C P$ violation. This feature of open-charm $B$ decays was first recognised in its application to $C P$ eigenstate decays of the $D[7,8]$ but was later extended to flavour-specific states accessible to both the 
$D^{0}$ and $\bar{D}^{0}$. This second category, labelled " $A D S$ " modes in reference to the authors of $[9,10]$, requires the favoured $b \rightarrow c$ decay to be followed by a suppressed $D$ decay, and the suppressed $b \rightarrow u$ decay to precede a favoured $D$ decay. The amplitudes of such combinations are of similar magnitude and hence large interference may be expected.

Using the summer 2011 dataset, LHCb finds evidence for the $A D S$ mode, $B^{ \pm} \rightarrow\left[\pi^{ \pm} K^{\mp}\right]_{D} K^{ \pm}$using multi-variant discriminator to reject combinatoric backgrounds and PID information to discriminate against dangerous peaking backgrounds. The size of this peak relative to the favoured $B^{ \pm} \rightarrow$ $\left[K^{ \pm} \pi^{\mp}\right]_{D} K^{ \pm}$mode is $R_{A D S}$. The charge asymmetry $A_{A D S}$. These variables are found to be

$$
\begin{aligned}
& R_{A D S}=(1.66 \pm 0.39 \pm 0.24) \times 10^{-2} \\
& A_{A D S}=-0.39 \pm 0.17 \pm 0.02
\end{aligned}
$$

which is of similar significance to the world best published results [11]. The invariant mass distribution of $B^{ \pm}$candidates is shown in Fig. 1 [12] which shows a peak of $4.0 \sigma$ total significance when compared to the null hypothesis.

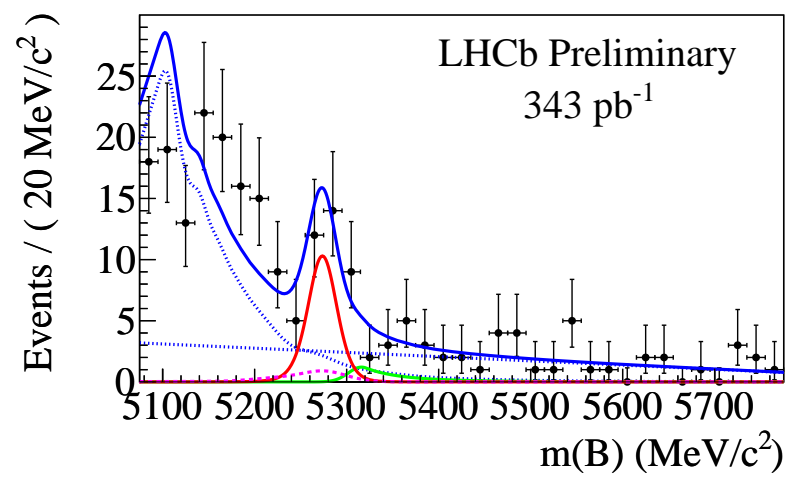

Fig. 1. The invariant mass distribution of $B^{ \pm} \rightarrow\left[\pi^{ \pm} K^{\mp}\right]_{D} K^{ \pm}$ candidates. The dashed line indicates the charmless background component. The light [green] shape is misidentified $B^{ \pm} \rightarrow$ $\left[\pi^{ \pm} K^{\mp}\right]_{D} \pi^{ \pm}$. The dotted lines are combinatoric and partially reconstructed backgrounds.

$2.2 B_{s}^{0} \rightarrow D_{s}^{\mp} K^{ \pm}$

The precision with which $\mathrm{LHCb}$ is able to resole secondary vertices has allowed precise measurements of $B_{s}^{0}$ oscillations [13] in excellent agreement with previous experiments [14]. Using this capability, time-dependent $C P$ violation effects may be studied, notably $\phi_{s}[15,16]$. With $\phi_{s}$ becoming well-known and converging on the Standard Model expectation, it becomes a small correction in rarer modes where $C P$ violation effects are expected to be larger. The leading such decay is $B_{s}^{0} \rightarrow D_{s}^{\mp} K^{ \pm}$which can be used to access $\gamma$ via the interference of $b \rightarrow c$ and $b \rightarrow u$ decays. The first step reported here, has been to confirm the signal mode with the summer 2011 dataset and perform a precise branching fraction measurement [17]. The signal peak is shown in Fig. 2 from which the following branching fraction measurement is deduced:

$$
\mathcal{B}\left(B_{s}^{0} \rightarrow D_{s}^{\mp} K^{ \pm}\right)=\left(1.97 \pm 0.18{ }_{-0.20}^{+0.19}{ }_{-0.10}^{+0.11}\right) \times 10^{-4}
$$

where the first uncertainty derives from the statistical uncertainty of the fit, the second from systematic effects and the third from the use of the fragmentation ratio $f_{s} / f_{d}$ in the normalisation.
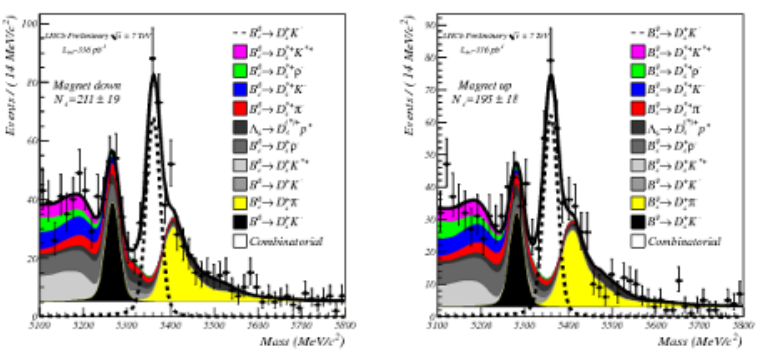

Fig. 2. The $B_{s}^{0}$ invariant mass distributions showing the $B_{s}^{0} \rightarrow$ $D_{s}^{\mp} K^{ \pm}$signal (dashed histogram). Background components are listed in the legend. The plot separates the summer 2011 samples by the polarity of the $\mathrm{LHCb}$ dipole.

\section{$2.3 \bar{B}_{s}^{0} \rightarrow D^{0} K^{* 0}$}

Self-tagging $\bar{B}^{0} \rightarrow D K^{* 0}$ decays offer similar sensitivity to $\gamma$ as the $B^{ \pm} A D S$ decay discussed above. However, this mode is rare and, in contrast to searches performed at the $B$ factories, suffers a background from $\bar{B}_{s}^{0}$ decays. Therefore the first step is to assess the potential problem from kinematically similar $B_{s}^{0}$ decays by searching for the Cabibboallowed, $\bar{B}_{s}^{0} \rightarrow D^{0} K^{* 0}$ mode. This has been completed [18] using the 2010 dataset and a significant peak is observed, see Fig. 3 leading to a branching fraction measurement of

$$
\mathcal{B}\left(\bar{B}_{s}^{0} \rightarrow D^{0} K^{* 0}\right)=(4.72 \pm 1.07 \pm 0.48 \pm 0.37 \pm 0.74) \times 10^{-4}
$$

where the first error is statistical, the second systemaic, the third from the branching fraction of the normalisation mode, $\bar{B}^{0} \rightarrow D^{0} \rho^{0}$ and the fourth from the ratio of $b \bar{b}$ fragmentation, $f_{s} / f_{d}$.

\section{$2.4 B^{-} \rightarrow D^{0} K^{-} \pi^{+} \pi^{-}$}

LHCb has recently developed the analysis of high multiplicity, $B \rightarrow D \pi \pi \pi$ decays [19]. These are experimentally 


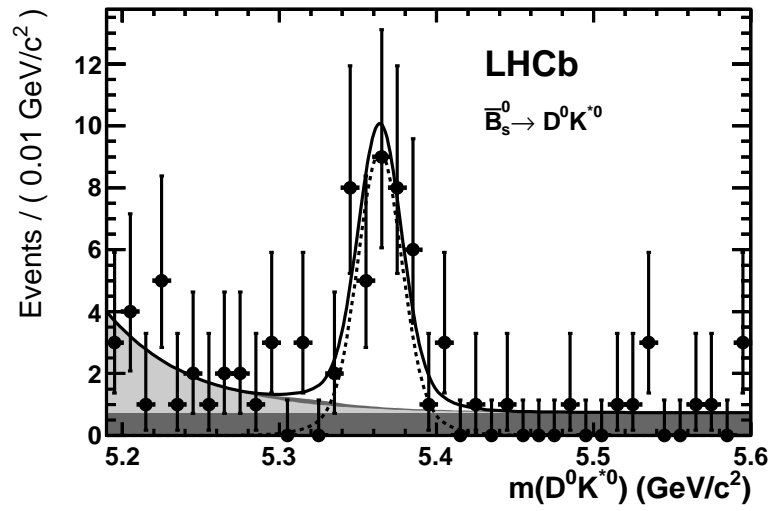

Fig. 3. The clear shape indicates the $\bar{B}_{s}^{0} \rightarrow D^{0} K^{* 0}$ signal on the 2010 sample; the light grey is partially reconstructed background and the dark shade is a combinatoric component.

challenging but will, in time, exhibit $\gamma$ sensitivity similar to simpler modes like $A D S$ mode discussed above. The first step has been to establish the favoured, and $\gamma$-insensitive $B^{\mp} \rightarrow D^{0} K^{\mp} \pi^{+} \pi^{-}$mode that will eventually be used as a control for rarer and more sensitive modes. Fig. 4 shows the clear mass peak accumulated with the data collected in 2010. The statistical significance of this peak is $8.0 \sigma$. This figure also shows the first observation of the topologically similar $B^{0} \rightarrow D^{\mp} K^{ \pm} \pi^{+} \pi^{-}$which has a significance of $6.6 \sigma$ [20].
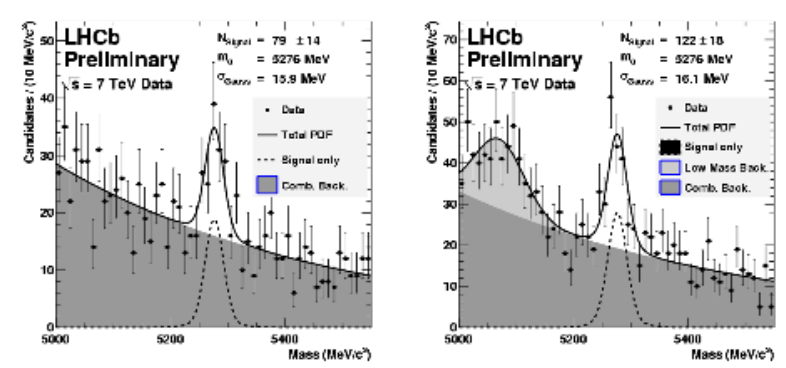

Fig. 4. left: $B^{0} \rightarrow D^{\mp} K^{ \pm} \pi^{+} \pi^{-}$, right: $B^{\mp} \rightarrow D^{0} K^{\mp} \pi^{+} \pi^{-}$. The description of the components maybe found in the legend.

$$
2.5 \Lambda_{b} \rightarrow p D^{0} K^{-}
$$

Few $b$-baryon decay modes have been observed and in those that have, no $C P$ violation is expected, nor observed. However, $\Lambda_{b}^{0}$ decays involving neutral $D$ mesons hold potential $\gamma$ sensitivity, analogous to the self-tagging $\bar{B}^{0} \rightarrow$ $D K^{* 0}$ mode mentioned above. The low fragmentation ratio for baryons compared to mesons, and the lower branching fractions to $D^{0}$ mesons means such an analysis is somewhat in the future. Nevertheless, LHCb has made an important step in establishing the eventual control mode $\Lambda_{b}^{0} \rightarrow$ $p D^{0} K^{-}$(charge conjugation implied). Its partial width with respect to that of the Cabibbo favoured $\Lambda_{b}^{0} \rightarrow p D^{0} \pi^{-}$is measured [21] as

$$
\frac{\mathcal{B}\left(\Lambda_{b}^{0} \rightarrow p D^{0} K^{-}\right)}{\mathcal{B}\left(\Lambda_{b}^{0} \rightarrow p D^{0} \pi^{-}\right)}=0.112 \pm 0.019_{-0.014}^{+0.011} .
$$

The invariant mass resolution distributions are shown in Fig. 5 which also shows a $2.6 \sigma$ hint of the neutral beautystange baryon decay, $\Xi_{b}^{0} \rightarrow p D^{0} K^{-}$around $5790 \mathrm{MeV} / c^{2}$.
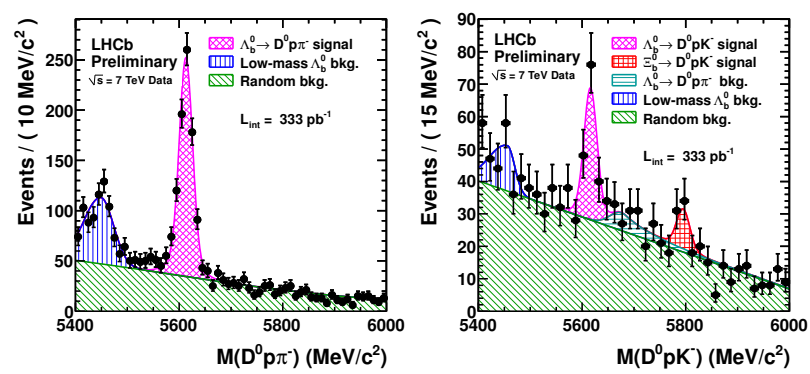

Fig. 5. left: $\Lambda_{b}^{0} \rightarrow p D^{0} \pi^{-}$, right: $\Lambda_{b}^{0} \rightarrow p D^{0} K^{-}$. The various components are described in the legend.

\section{Searches for CP violation in charm}

This section reports the searches for $C P$ violation in the charm sector using the data collected in 2010.

\subsection{CP violation in charm mixing}

Like any neutral meson system, the interacting weak eigenstates, $\left|D_{1,2}\right\rangle$, can be represented as a linear sum of the mass eigenstates: $\left|D^{0}\right\rangle,\left|\bar{D}^{0}\right\rangle$. The mass and lifetime differences between $D_{1}$ and $D_{2}$,

$$
\begin{aligned}
& x=\left(m_{2}-m_{1}\right) / 2 \Gamma, \\
& y=\left(\Gamma_{2}-\Gamma_{1}\right) / 2 \Gamma
\end{aligned}
$$

are the mixing parameters whose non-zero values have demonstrated $D^{0}$ mixing [5]. Searches for $C P$ violation can be made by looking for differences in the mixing parameters in $C P$, and non- $C P$ modes. $\mathrm{LHCb}$ does not find evidence of $C P$ violation by this method and reports [22]

$$
\begin{aligned}
y_{C P} & =\frac{\Gamma\left(D^{0} \rightarrow K^{+} K^{-}\right)}{\Gamma\left(D^{0} \rightarrow K^{-} \pi^{-}\right)}-1 \\
& =(5.5 \pm 6.3 \pm 4.1) \times 10^{-3}
\end{aligned}
$$

in agreement with the world average: $(1.11 \pm 0.22) \%$. 
Another useful observable used to probe $C P$ violation is $A_{\Gamma}$, the difference in lifetime of $D^{0}$ and $\bar{D}^{0}$ to $C P$ eigenstates. This measurement is similar to the $y_{C P}$ analysis, separating the prompt $D^{0}$ decays from the component coming from $B$ decays using a fit to the impact parameter distribution. Also, a data-driven technique is employed to estimate the lifetime biases in the trigger selection. From the 2010 dataset, $\mathrm{LHCb}$ measures

$$
\begin{aligned}
A_{\Gamma} & =\frac{\Gamma\left(D^{0} \rightarrow K^{+} K^{-}\right)-\Gamma\left(\bar{D}^{0} \rightarrow K^{-} K^{-}\right)}{\Gamma\left(D^{0} \rightarrow K^{+} K^{-}\right)+\Gamma\left(\bar{D}^{0} \rightarrow K^{-} K^{-}\right)} \\
& =(-5.9 \pm 5.9 \pm 2.1) \times 10^{-3}
\end{aligned}
$$

in agreement with the world average of $(0.12 \pm 0.25) \%$.

\subsection{Direct CP violation in charm decays}

Singly Cabibbo-suppressed, multi-body $D$ decays may manifest an effective $C P$ violation up to the $1 \%$ level in certain new physics models. $\mathrm{LHCb}$ chooses to search for such effects in a model-independent manner by considering charge asymmetries in 2D bins of various sizes across the Dalitz plot of $D^{ \pm} \rightarrow K^{+} K^{-} \pi^{ \pm}$decays. One of the four binning schemes investigated is shown in Fig. 6. With such a method one expects, if no $C P$ violation is present, that the distribution of the $\mathrm{N}$ measured charge asymmetries (from $\mathrm{N}$ bins) is distributed according to a Gaussian function. Whereas the occurrence of $C P$ violation in some unspecified region of the Dalitz plot would appear as a bias or a tail in such a distribution. Using a sample of $3.7 \times 10^{5} D^{ \pm} \rightarrow K^{+} K^{-} \pi^{ \pm}$ decays from 2010, no hint of $C P$ violation is yet seen [23].

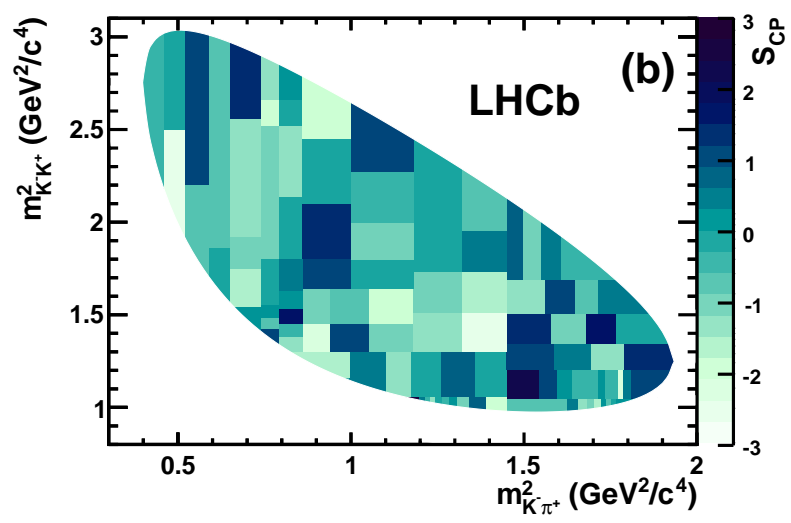

Fig. 6. One of the binning schemes used in the modelindependent search for direct $C P$ violation in charm.

\section{Acknowledgements}

The speaker wishes to thank the organisers of HCP2011 for an excellent conference and to his LHCb collaborators who produces the individual results described here.

\section{References}

1. N. Cabibbo, Phys. Rev. Lett. 10, 531 (1963)

2. M. Kobayashi, T. Maskawa, Prog. Theor. Phys. 49, 652 (1973)

3. B. Aubert et al. (BABAR), Phys. Rev. Lett. 87, 091801 (2001), hep-ex/0107013

4. K. Abe et al. (Belle Collaboration), Phys.Rev.Lett. 87, 091802 (2001), hep-ex/0107061

5. D. Asner et al. (Heavy Flavor Averaging Group) (2010), hep-ex/1010.1589

6. A.A. Alves Jr. et al. (LHCb collaboration), JINST 3, S08005 (2008)

7. M. Gronau, D. London, Phys. Lett. B253, 483 (1991)

8. M. Gronau, D. Wyler, Phys. Lett. B265, 172 (1991)

9. D. Atwood, I. Dunietz, A. Soni, Phys.Rev.Lett. 78, 3257 (1997), hep-ph/9612433

10. D. Atwood, I. Dunietz, A. Soni, Phys.Rev. D63, 036005 (2001), hep-ph/0008090

11. Y. Horii et al. (Belle), Phys. Rev. Lett. 106, 231803 (2011), hep-ex/1103. 5951

12. R. Aaij et al. (LHCb Collaboration) (2011), see LHCbCONF-2011-044

13. R. Aaij et al. (LHCb Collaboration) (2011), hep-ex/1112. 4311

14. A. Abulencia et al. (CDF Collaboration), Phys. Rev. Lett. 97, 062003 (2006)

15. R. Aaij et al. (LHCb Collaboration) (2011), hep-ex/1112. 3183

16. R. Aaij et al. (LHCb Collaboration), Phys.Lett. B707, 497 (2012), long author list - awaiting processing, hep-ex/1112. 3056

17. R. Aaij et al. (2011), see LHCb-CONF-2011-057

18. R. Aaij et al. (LHCb Collaboration), Phys.Lett. B706, 32 (2011), hep-ex/1110. 3676

19. R. Aaij et al. (LHCb Collaboration), Phys.Rev. D84, 092001 (2011), hep-ex/1109.6831

20. R. Aaij et al. (LHCb Collaboration) (2012), hep-ex/1201.4402

21. R. Aaij et al. (2011), see LHCb-CONF-2011-036

22. R. Aaij et al. (LHCb Collaboration) (2011), hep-ex/1112.4698

23. R. Aaij et al. (LHCb Collaboration), Phys.Rev. D84, 112008 (2011), hep-ex/1110. 3970 